\title{
Resonant Neutrino Interaction and Mixing
}

\author{
G. Domokos and S. Kovesi-Domokos \\ INFN, Sezione di Firenze \\ Florence, Italy \\ and
}

The Henry A. Rowland Department of Physics and Astronomy

The Johns Hopkins University

Baltimore, MD 21218円

We investigate the resonant interaction of neutrinos in matter in the presence of mixing. At energies near the $\mathrm{W}$ resonance, oscillations are absent; the survival probability of electron antineutrinos is suppressed due to the presence of open inelastic channels.

The existence of a resonant interaction of electron antineutrinos in matter and the possibility of flavor oscillations have been known for a long time; for a summary see [1]. The laboratory energy at which the resonance occurs is about $6.4 \mathrm{PeV}$. Recently, interest has turned towards an investigation of neutrino oscillations in the $\mathrm{PeV}$ energy range, at energies near the $\mathrm{W}$ resonance in the $\bar{\nu}_{e} e$ interaction cross section [2]. Learned and Pakvasa in ref. [2] point out that neutrino oscillations in a hitherto unexplored range of neutrino masses and mixing angles may lead to the arrival and detection of $\tau$ neutrinos in present and planned neutrino telescopes (DUMAND, NESTOR, KM3 etc. ).

It is generally assumed [3] that neutrinos emerging from a celestial source (typically, an AGN or a binary system) are produced as a result of the decay of hadrons. If so, in either hadron-hadron interactions or in photoproduction most of the hadrons produced are pions, with charged pions of either sign being produced in approximately equal numbers and energy distributions. Consequently, the number of neutrinos and antineutrinos of any flavor is approximately equal. To a reasonable approximation, the ratio of neutrinos (antineutrinos, resp.) of different flavors produced is:

$$
\nu_{e}: \nu_{\mu}: \nu_{\tau} \approx 1: 2: 0
$$

It has to be noted, however that at least some of the time, a considerable amount of matter is present between the primary source of neutrinos and the detectors in the experiments mentioned.

- In all probability, neutrinos produced in an AGN or a binary system, have to pass through a region of high electron (and perhaps baryon) density.

- In addition, the neutrinos usually pass through a certain amount of matter between the source and the detector, e.g. the Earth (in the case of the observation of upward going neutrinos) and/or sea water (in case of zenith angles close to the horizon).

\footnotetext{
${ }^{1}$ Permanent address. E-mail: SKD @.JHUP.PHA.JHU.EDU
} 
In this note we begin to investigate the question of neutrino oscillations if neutrinos in the PeV energy range (around the energy of where the $\mathrm{W}$ resonance occurs) penetrate matter between the source and the detector. Our main purpose here is to explore the qualitative aspects of the phenomena. For this reason, we use the formalism developed by Wolfenstein [4] and a hypothetical situation of having two flavors only. Furthermore, the medium is assumed to be a homogeneous one.

A full treatment taking into account all three flavors and a more complete treatment of the presence of inelastic channels and inhomogeneities in the medium will be given elsewhere.

The Wolfenstein equation can be viewed as a Schrödinger equation in a null plane formalism. (It has been known for a long time that the kinematics in the two dimensional transverse space is Galilean; for a description of the formalism with an emphasis on its group theoretical aspects, see [5, 6], particularly the second of these references.)

The behavior of the evolution operator of neutrinos, $E$, is governed by the equation:

$$
i \partial_{t} E=\frac{\left(\vec{P}^{2}+M^{2}+V\right)}{2 p} E,
$$

where $p$ and $\vec{P}$ stand for the longitudinal and transverse components of the momentum, respectively; $M^{2}$ and $V$ are the mass squared and interaction operators. The variable $t$ may be thought of as the time conjugate to the Hamiltonian,

$$
H=\frac{\vec{P}^{2}+M^{2}+V}{2 p} .
$$

The evolution operator as well as the mass squared, interaction and transverse momentum operators are to be thought of as $f \times f$ dimensional matrices, where $f$ is the number of flavors. The operator $\vec{P}$ is proportional to the unit matrix. The important feature of the null plane formalism is that the longitudinal and transverse degrees of freedom are almost exactly separated. Thus, $p$ may be diagonalized separately and quantities like $V$, governing the dynamics in the transverse plane may depend on its eigenvalue. (For the same reason, $p$ is, to a good approximation, equal to the kinetic energy of the neutrinos at the source.)

In the conventional treatment of neutrino oscillations (for a review, see [7]) one omits the square of the transverse momentum and represents the matter present in terms of a potential independent of the transverse degrees of freedom. (This is equivalent to the classic Lorentz formula, expressing the polarizability of a medium in terms of the forward scattering amplitude of light on the particles in the medium.)

Since we are to treat a resonant interaction, such an approximation is not an adequate one. We can still neglect the transverse degrees of freedom; however, a more accurate expression has to be found for the "potential". The appropriate 
procedure has been known for a long time, see [8] and the original references quoted there.

Briefly, the recipe is that wherever a potential appears in the the lowest order treatment of the scattering, it has to be replaced by an effective potential. The latter is given by the level shift operator (the " $K$ matrix") of the scattering of the propagating particle in the medium. The relationship between the transition operator, $\mathbf{T}$ and the $\mathbf{K}$ matrix is:

$$
\mathbf{T}=-\mathbf{K}(1+i \mathbf{K})^{-1}
$$

We refer the reader to Brueckner's lecture in ref. [8] for a derivation of the "recipe" just quoted. However, we can make it plausible by recalling that the $K$ matrix obeys the Lippmann-Schwinger equation. In an operator form the latter reads:

$$
\mathbf{K}=\mathbf{V}+\mathbf{V} \frac{\mathcal{P}}{\epsilon-\mathbf{H}_{0}} \mathbf{K}
$$

where $\epsilon$ is the energy (in the present context, $\epsilon=p$ ) and $\mathbf{H}_{0}$ is the free Hamiltonian, see e.g. ref. [9]. The symbol of the principal value, $\mathcal{P}$, simply means that those eigenstates of the free Hamiltonian for which the inverse of $\epsilon-H_{0}$ is singular, are to be deleted from a complete set. One now realizes that the first term in the iterative solution of eq. (3) is indeed the potential; moreover, in the absence of open inelastic channels, the operator $\mathbf{K}$ is a Hermitean one, thus it qualifies as a "potential" in a Schrödinger equation.

Armed with this knowledge, we can now construct a phenomenological effective potential describing the resonant interaction of electron antineutrinos in matter. We describe the scattering amplitude (the matrix element of $\mathbf{T}$ ) by means of a Breit-Wigner formula; the forward amplitude is given by:

$$
T=\frac{s \mathrm{M}^{2} G_{F} \sqrt{2} / 6 \pi}{\mathrm{M}^{2}-s-i \mathrm{M} \Gamma_{t}} .
$$

In the last equation, $M$ stands for the mass of the W, $\Gamma_{t}$ is its total width. $G_{F}$ stands for the conventional Fermi coupling constant and $s$ is the square of the center of mass energy. In terms of the mass of the electron and $p$ it is given by $s=2 m_{\mathrm{e}} p$. We note that in terms of these quantities, the elastic width of the $\mathrm{W}$ is given by the expression, $\Gamma_{e}=M^{3} G_{F} / 6 \pi \sqrt{2}$. In what follows, we take the numerator of eq. (4) at $s=M^{2}$; this is a good approximation for a narrow resonance. Due to the fact that we neglect all but the resonant partial wave in eq. (四), the expression of the matrix element of the $\mathbf{K}$ matrix is found immediately, viz.

$$
K=-\frac{M \Gamma_{e}}{\mathrm{M}^{2}-s-i M \Gamma_{i}}
$$

where $\Gamma_{i}=\Gamma_{t}-\Gamma_{e}$ is the inelastic width of the W. If the inelastic width, $\Gamma_{i}=$ $\Gamma_{t}-\Gamma_{e}$ were zero, the expression in eq. (5) would be real as required. Thus the 
effective potential in the medium entering eq. (四) is given by the expression,

$$
V_{e f f}=\frac{n_{\mathrm{e}}}{m_{\mathrm{e}}} K
$$

where $n_{\mathrm{e}}$ is the electron density in the medium.

In the present model, the flavor space is two dimensional; we denote the flavors by e and $\mu$, respectively. Due to the fact that (in real life), both the electron and muon are much lighter than the $\mathrm{W}$, the matrix elements of the effective potential, $\mathcal{F}$ are:

$$
\langle\mu|\mathcal{F}| \mathrm{e}\rangle=\langle\mathrm{e}|\mathcal{F}| \mathrm{e}\rangle=F
$$

and all other matrix elements vanish.

Next, we give the matrix elements of the mass squared matrix, $\mathcal{M}^{2}$ :

$$
\begin{aligned}
\left\langle e\left|\mathcal{M}^{2}\right| \mathrm{e}\right\rangle & =-\left\langle\mu\left|\mathcal{M}^{2}\right| \mu\right\rangle \\
& =\Delta^{2} \cos 2 \Theta \\
\left\langle\mu\left|\mathcal{M}^{2}\right| \mathrm{e}\right\rangle & =\left\langle\mathrm{e}\left|\mathcal{M}^{2}\right| \mathrm{e}\right\rangle \\
& =\Delta^{2} \sin 2 \Theta
\end{aligned}
$$

As usual, we subtracted the average mass squared; its presence merely multiplies the state vectors by an overall phase factor, hence, it has no influence on neutrino oscillations.

We now have to solve eq. (11) with the effective Hamiltonian put together from eqs. (8) and (7). Despite the fact that the Hamiltonian is not a Hermitean matrix in flavor space, the equation can be solved immediately. This is due to the fact that any non singular $2 \times 2$ matrix can be diagonalized by means of a similarity transformation. (If the matrix in question is not a Hermitean one, the diagonalizing matrices are elements of the group $\mathrm{SL}(2, \mathrm{C})$ instead of $\mathrm{SU}(2)$.) We merely quote the result of the calculation.

$$
\begin{aligned}
\langle\mathrm{e}|E| \mathrm{e}\rangle & =\exp \left(-i t \frac{F}{4 p}\right)\left[\cos \phi-i \sin \phi \frac{\Delta^{2} \cos 2 \Theta+F / 2}{\mu^{2}}\right] \\
\langle\mu|E| m u\rangle & =\exp \left(-i t \frac{F}{4 p}\right)\left[\cos \phi+i \sin \phi \frac{\Delta^{2} \cos 2 \Theta+F / 2}{\mu^{2}}\right] \\
\langle\mu|E| \mathrm{e}\rangle & =-i \exp \left(-i t \frac{F}{4 p}\right) \sin \phi \frac{\Delta^{2} \sin 2 \Theta+F}{\mu^{2}} \\
\langle e|E| \mu\rangle & =-i \exp \left(-i t \frac{F}{4 p}\right) \sin \phi \frac{\Delta^{2} \sin 2 \Theta}{\mu^{2}}
\end{aligned}
$$

The quantities $\mu^{2}$ and $\phi$ entering these equations are defined as follows.

$$
\begin{aligned}
\mu^{2} & =\left(\Delta^{4}+F \Delta^{2}(\cos 2 \Theta+\sin 2 \Theta)+F^{2} / 4\right)^{1 / 2} \\
\phi & =\frac{t \mu^{2}}{2 p}
\end{aligned}
$$


The physical interpretation of these equations is not quite easy, due to the fact that the quantity $\mathrm{F}$ is complex. However, near the resonance itself (where the use of the Breit-Wigner formula is justified in the first place), considerable simplifications occur. In order to understand this, we notice that the quantity $n_{\mathrm{e}} / m_{\mathrm{e}}$ ocurring in the definition of the effective potential, defines a mass scale characterizing the medium. In the following Table we exhibit approximate values of the mean electron density, together with the characteristic mass scale, $\mathrm{m}_{c}^{2}=n_{\mathrm{e}} / m_{\mathrm{e}}$ for some environments of interest.

\section{Electron densities and characteristic masses for some environments}

\begin{tabular}{|c|c|c|}
\hline Environment & $n_{\mathrm{e}}\left[\mathrm{cm}^{-3}\right]$ & $\mathrm{m}_{c}^{2}\left[\mathrm{eV}^{2}\right]$ \\
\hline stellar interior (sun) & $10^{27}$ & $\left.2 \times 10^{7}\right]$ \\
Earth & $1.6 \times 10^{24}$ & $3 \times 10^{4}$ \\
water & $3 \times 10^{23}$ & $5 \times 10^{3}$ \\
\hline
\end{tabular}

We see that the characteristic mass scales are, in general, considerably larger than the expected values of $\Delta^{2}$ in a typical mixing scheme occurring in the literature, see, for instance, [1] for a review. As a consequence, the expression of $\mu^{2}$ is considerably simplified near $s=M^{2}$. In fact, we have:

$$
\mu^{2} \approx \frac{F}{2}+\Delta^{2}(\sin 2 \Theta+\cos 2 \Theta)
$$

so that to leading order, $\mu^{2} \approx F / 2$. Using the Euler decomposition of the trigonometric functions, one realizes that in the terms proportional to

$$
\exp (-i t F / 4 p) \exp i \phi
$$

$F$ cancels out to a good approximation. By contrast, in terms proportional to

$$
\exp (-i t F / 4 p) \exp -i \phi
$$

$F$ is present in the exponent. The quantity $F$ has an imaginary part due to the fact that there are inelastic channels open: W decays mostly hadronically. Therefore terms proportional to $\exp (-i F t / 2 p)$ are exponentially damped and all surviving terms in the expression of the transition matrix have the same phase. (By putting in numbers one concludes that the extinction distance is a few metres at energies near the resonance in any environment of interest; thus, at any reasonable value of $\Delta^{2}$ the damped terms are negligible.) Consequently, we omit terms proportional to $\exp (-\operatorname{Im} F t)$ in what follows.

We now use the approximation introduced in eq. (10) to give the expressions of the transition probabilities. This is best done by introducing the dimensionless distance in energy from the resonance, $x$ and an appropriately scaled ratio of $\Delta^{2} / m_{c}^{2}$ : 


$$
\begin{aligned}
& x=\frac{s-M^{2}}{M \Gamma_{i}} \\
& \delta=\frac{\Delta^{2} \Gamma_{i}}{m_{c}^{2} \Gamma_{e}}
\end{aligned}
$$

The transition probabilities are then given by the expressions:

$$
\begin{aligned}
|\langle e|E| e\rangle|^{2} & =\frac{(\delta \sin 2 \Theta)^{2}}{1+\left(x+2 \delta(\sin 2 \Theta+\cos 2 \Theta)\left(1+x^{2}\right)\right)^{2}} \\
|\langle\mu|E| e\rangle|^{2} & =\frac{1+\left(x+\delta \sin 2 \Theta\left(1+x^{2}\right)\right)^{2}}{1+\left(x+2 \delta(\sin 2 \Theta+\cos 2 \Theta)\left(1+x^{2}\right)\right)^{2}} \\
|\langle\mu|E| \mu\rangle|^{2} & =\frac{1+\left(x+\delta \sin 2 \Theta\left(1+x^{2}\right)\right)^{2}}{1+\left(x+2 \delta(\sin 2 \Theta+\cos 2 \Theta)\left(1+x^{2}\right)\right)^{2}} \\
|\langle e|E| \mu\rangle|^{2} & =\frac{(\delta \sin 2 \Theta)^{2}\left(1+x^{2}\right)^{2}}{1+\left(x+2 \delta(\sin 2 \Theta+\cos 2 \Theta)\left(1+x^{2}\right)\right)^{2}}
\end{aligned}
$$

By looking at the last set of equations, one realizes that the survival probability of electron antineutrinos is very low; this is due to the fact that the $\mathrm{W}$ resonance is a highly inelastic one: once the electron antineutrino interacts, the $\mathrm{W}$ decays into the original channel only rarely. By contrast, the muon antineutrinos are hardly depleted and they hardly ever produce electron antineutrinos: these processes proceed through mixing only. Remarkably, the $t$-dependence of all transition probabilities disappears beyond the extinction distance: an inelastic resonance produces uniform transition probabilities.

Let us summarize.

- The presence of an inelastic resonance stongly suppresses oscillation effects.

- The original 1:2:0 ratio of the antineutrinos of the three flavors is strongly distorted. Electron antineutrinos are depleted, while muon antineutrinos are hardly affected. (In fact, their number is slightly increased due to the decay $\mathrm{W} \rightarrow \mu \bar{\nu}_{\mu}$.)

- One can reasonably conjecture that in a calculation taking three generations into account, $\bar{\nu}_{\tau}$ production will be comparable to the $\bar{\nu}_{\mathrm{e}} \rightarrow \bar{\nu}_{\mu}$ rate (with the appropriate mixing angles and mass differences), i.e. a considerable number of $\tau$ leptons and the corresponding antineutrinos will be produced. In particular, the appearence of $\bar{\nu}_{\tau^{-S}}$ in a neutrino telescope does not necessarily imply oscillation lengths comparable to the distance to some AGN.

- The ratios of neutrinos (as opposed to antineutrinos) should be unaffected unless there exist neutrino-antineutrino oscillations. 


\section{Acknowledgement}

This research has been supported in part by the U.S. Department of Energy under Grant \# DE-FG-02-85ER40211.

We also thank Roberto Casalbuoni, John Learned and Leon Madansky for enlightening conversations on the subject.

Most of this work has been done during the authors' visit at the Istituto di Fisica, Universitá di Firenze, during the Summer of 1994. We thank Roberto Casalbuoni for his hospitality and the INFN for partial financial support.

\section{References}

[1] C.W. Kim and A. Pevsner, "Neutrinos in Physics and Astrophysics". Harwood Academic Publishers, Chur, Switzerland (1993).

[2] J.G. Learned and S. Pakvasa, preprint UH-511-799-94; to be published in Astroparticle Physics.

[3] See the Proceedings of the Hawaii Conference on High Energy Neutrino Astrophysics for an overview of the "state of the art". (Edited by J.G. Learned, S. Pakvasa, V.J. Stenger and X. Tata.) World Scientific Publishing Corp. Singapore (1992)

[4] L. Wolfenstein, Phys. Rev. D 17 (1978) 2360.

[5] G. Domokos, Phys. Rev. D 4 (1971) 3708

[6] G. Domokos, in "Lectures on Theoretical Physics", Vol. XIV. Edited by A.O. Barut and W.E. Brittin. University of Colorado Press, Boulder, CO (1972).

[7] T.K. Kuo and J. Pantaleone, Rev.Mod.Phys. 61 (1989) 937.

[8] K.A. Brueckner in "Le Problem a N Corps" (Proceedings of the 1957 Les Houches Summer School). Edited by C. Morette-DeWitt. Dunod, Paris (1958).

[9] M.L. Goldberger and K.A. Watson, "Collision Theory". Wiley, New York (1964). 